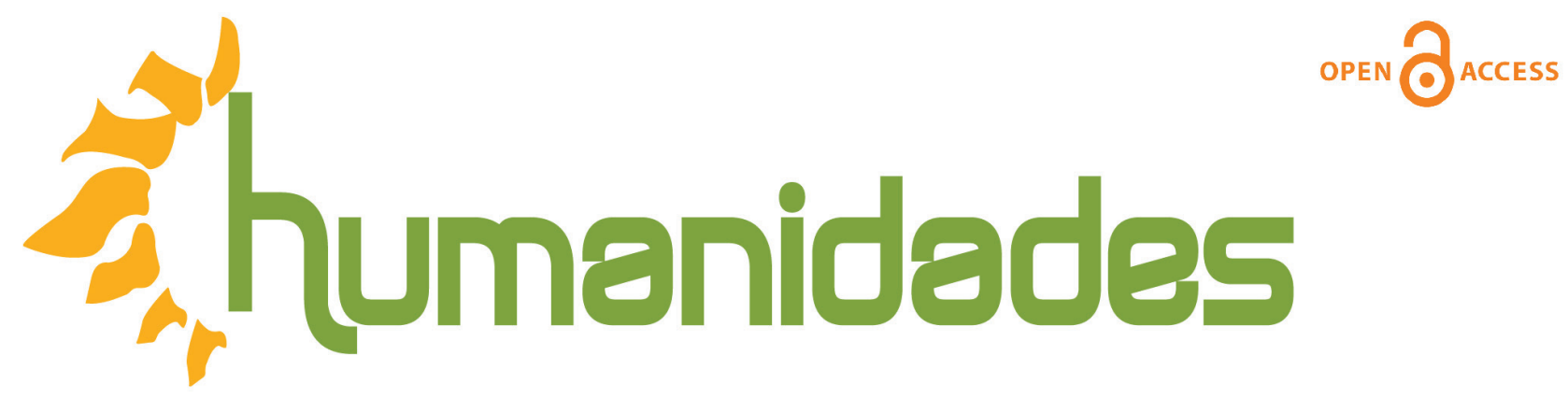

Revista de la Escuela de Estudios Generales, Universidad de Costa Rica

Julio-diciembre, 2019 • Volumen 9, número 2 • EISSN 2215-3934 • pp. 1-6

\title{
Reflexiones desde la academia. Universidad, Ciencia y Sociedad
}

DOI: https://doi.org/10.15517/h.v9i2.37657

\section{Alexander Jiménez Matarrita}

Doctor, profesor catedrático

Escuela de filosofía, Universidad de Costa Rica, Costa Rica

Correo electrónico: jimenezm@usal.es / nicolae.orescu@gmail.com

Todos los derechos reservados. Universidad de Costa Rica. Esta revista se encuentra licenciada con Creative Commons. Reconocimiento-NoComercial-SinObraDerivada 3.0 Costa Rica. Correo electrónico: humanidades@ucr.ac.cr/ Sitio web: http: //revistas.ucr.ac.cr/index.php/ humanidades 


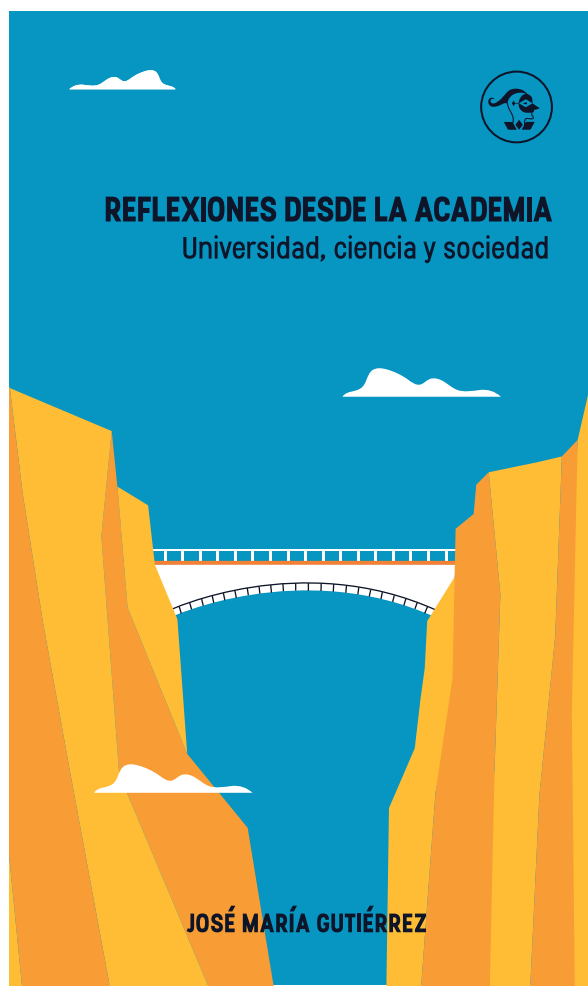

Reflexiones desde la academia.

Universidad, Ciencia y Sociedad, San José, Costa Rica,

Editorial Arlekin, 2018, 385 págs.
Reflexiones desde la academia. Universidad, Ciencia y Sociedad, de José María Gutiérrez, es un libro propicio para entender el lugar central que tienen las universidades públicas y las ciencias en el desarrollo humano de nuestras sociedades.

Uno de los supuestos que sostienen las argumentaciones de Gutiérrez es que el conocimiento y la investigación son condiciones necesarias para formas de convivencia justas, solidarias, generosas. Una buena parte de su libro está dedicada a demostrar ese vínculo virtuoso.

Reflexiones desde la academia. Universidad, Ciencia y Sociedad es también una poderosa defensa de las universidades públicas y las ciencias frente a proyectos empeñados en impedir sus condiciones habituales de existencia $\mathrm{y}$, aún, su misma existencia. La superstición y la lógica mercantil, basada en el puro cálculo de utilidad, son modos de poner en riesgo dichas instituciones. Ante dichos desafíos, el desarrollo del conocimiento y de la investigación sigue siendo una exigencia de las universidades públicas. 
El libro de Gutiérrez permite comprender los complejos escenarios en medio de los cuales las ciencias y las universidades experimentan distintos tipos de asedio y aversión. Al mismo tiempo, se trata de un texto que pone en cuestión, es cierto que no siempre en toda su amplitud, las respuestas de dichas instituciones ante esos escenarios.

De esta manera, en los once capítulos del texto el autor discurre, desde su sólida experiencia académica y sus estudios científicos, por el universo que constituyen las relaciones entre la universidad, la ciencia y la sociedad. A pesar de que el título no parezca contenerlo, el texto también se ocupa de reflexionar en torno a un ámbito central de nuestra vida cotidiana: la tecnología.

Se trata de un texto cruzado por las reflexiones, agudas y sugerentes, de un científico quien es también un maestro y un ciudadano. Desde una mirada amplia y pluridisciplinar, Gutiérrez se empeña en pensar sobre cómo el conocimiento y la investigación son elementos imprescindibles para construir sociedades que hagan posible el desarrollo humano de sus habitantes.

A continuación se transcriben varios pasajes de la Introducción del libro Reflexiones desde la academia. Universidad, Ciencia y Sociedad, titulada "A manera de preámbulo". Esta introducción dibuja los asuntos sustantivos tratados en dicho libro. Escrito en primera persona, se trata de un breve texto en el cual también puede percibirse el lugar desde el cual Gutiérrez piensa sus asuntos.

Alexander Jiménez Matarrita

"La presente colección de ensayos recoge reflexiones de muchos años, generadas al calor de mis actividades docentes, de investigación y de acción social en la Universidad de Costa Rica (UCR) y de procesos diversos en los que me he involucrado. Aunque algunos de los tópicos tratados pueden parecer, a primera vista, un tanto heterogéneos, pretendo que los mismos tengan un hilo conductor, centrado en consideraciones sobre las universidades públicas, con énfasis en la generación de conocimiento mediante la investigación, y en la preocupación de cómo fortalecer el carácter público, humanista y de vocación académica de estas instituciones. 
Además, este hilo se prolonga con el tema de cómo la universidad y la ciencia pueden, y deben, contribuir con la construcción de formas de convivencia más generosas y solidarias.

Los ensayos se pueden leer de manera independiente, pues son o bien artículos publicados en varios medios y actualizados para esta obra, o textos elaborados a partir de participaciones en actividades académicas como foros y mesas redondas. En otros casos, los ensayos fueron preparados de novo para ser incluidos en esta colección. Aunque la gran mayoría de las reflexiones se basan en vivencias en la Universidad de Costa Rica, las mismas pueden extenderse, mutatis mutandis, a otras instituciones de educación superior públicas de la región latinoamericana.

La UCR, y las universidades públicas, en general en América Latina, están inmersas en un escenario complicado. Históricamente han sido instituciones claves en procesos culturales, sociales, políticos y económicos en nuestros países, constituyéndose, a la vez, vías de promoción social y de gestación de oportunidades, generadoras de nuevo conocimiento y núcleos de pensamiento innovador y crítico...

A partir de la década de 1980, la onda expansiva de los procesos de globalización neoliberal, con sus valores subyacentes, ha tenido un fuerte impacto en las universidades públicas. El debilitamiento progresivo del sector público y la promoción del concepto de la educación como un bien mercantil más que como un derecho básico de las personas, ha incidido de diferentes maneras en el mundo universitario latinoamericano. A ello se suma, en muchos países, el creciente estrangulamiento presupuestario, así como los intentos variados de cooptación de las universidades públicas para que se ajusten a la lógica del mercado. La emergencia del sector privado de educación superior, incluido el componente transnacional, ha complicado aún más un entorno ya de por sí desafiante. Las universidades, conjuntamente con otros sectores de las sociedades, han enfrentado estos retos con posicionamientos tendientes a fortalecer el ethos académico y filosófico que las fundamenta. Esta realidad compleja y multifactorial demanda de nuestras instituciones de educación superior esfuerzos intensos de análisis, discusión y transformación. 
Algunos de los ensayos que aquí se presentan buscan generar un debate crítico sobre la situación actual y futura de las instituciones de educación superior públicas, con énfasis en la Universidad de Costa Rica. Es preocupante que, precisamente cuando ocurren procesos que tienden a minar el estatus público de estas instituciones y a cuestionar aspectos centrales de lo que ha sido su misión, sea frecuente en nuestras comunidades universitarias una actitud de indiferencia ante los asuntos aquí tratados.

Un primer objetivo de esta publicación es, por lo tanto, colocar sobre el tapete del debate lo que considero son facetas fundamentales de las universidades en la actualidad, tales como la esencia del carácter público de las mismas, la necesidad de fortalecer y renovar la relación entre la universidad y diversos sectores de la sociedad, la relevancia de la formación humanista y solidaria, unida al respeto por el desarrollo integral de todos los ámbitos del conocimiento, y el mejoramiento de nuestro propio quehacer académico.

También se discute el tema de la investigación y de la necesidad de generar conocimiento endógeno como un elemento consustancial del patrimonio cultural de nuestros países, para comprender nuestra realidad natural y social, y para desarrollar insumos que permitan transformarla. Al calor de este desiderátum, se discute la relevancia del posgrado y del cultivo de entornos de creatividad en las universidades públicas, así como de elevar los niveles de exigencia a lo interno, y de implementar procesos de internacionalización centrados en el desarrollo académico, la cooperación y la solidaridad.

Los últimos ensayos se centran más en mi propio ámbito disciplinar, la Microbiología y las Ciencias Biomédicas, y exploran temas como la relación entre las ciencias naturales y las ciencias sociales, la historia de la Microbiología Médica, el legado de Clodomiro Picado Twight y la forma como Costa Rica ha enfrentado el problema de los envenenamientos por mordeduras de serpiente mediante un proyecto de carácter holístico e inter-institucional, con una fuerte proyección internacional. 
Aunque son tópicos más puntuales, los mismos pretenden ilustrar la importancia del trabajo inter- y trans-disciplinario y cómo el desarrollo de la ciencia y la tecnología tiene un profundo impacto en el entorno social en el que transcurren los esfuerzos académicos”. 